\title{
Live Demonstration: A Dynamically Adaptable Image Processing Application Running in an FPGA-Based WSN Platform
}

\author{
Alfonso Rodríguez, Juan Valverde, César Castañares, Jorge Portilla, Eduardo de la Torre and Teresa Riesgo
}

\section{INTRODUCTION AND BACKGROUND}

This 1-Page Demonstration paper is included in the track "Multimedia Systems and Applications". The work has been already published in [1] and [2]. The main idea of the demonstration is to show how the Virtual Architecture $\mathrm{ARTICo}^{3}$ works within a high performance wireless sensor node called HiReCookie. The selected demo includes an image processing application with several filters running as different kernels within the architecture $\mathrm{ARTICo}^{3}$. The virtual architecture works in a Spartan-6 FPGA included in the HiReCookie Node, [3] and [4]. During the demonstration, an image taken from a video camera attached to the node will be processed in real time by several dynamically reconfigurable kernels (median filters and edge detectors) under different working conditions. The solution scope includes solutions trading off among Low Power, Dependability and High Performance Computing.

\section{DEMONSTRATION SETUP}

The demonstration setup is shown in Fig. 1. The material required for the demo is listed below:

- Laptop running the software application.

- HiReCookie Node including the video camera.

- USB cable to power the node and send commands via UART.

- Ethernet switch to send the images from the node to the software application.

- Camera support to hold the camera in a fixed position.

\section{VISITOR EXPERIENCE}

During the demonstration of this work, the visitor will be able to see and interact with the system in the following ways:

- Both the original and processed images are shown in the SW application to observe changes in real time.

- It will be possible to change working conditions by sending commands to the node from the SW app and observe how processing time and power consumption change in real time.

- The internal changes in the virtual architecture (through Dynamic and Partial Reconfiguration) corresponding to each operation mode are shown in real time to fully understand the demonstration.

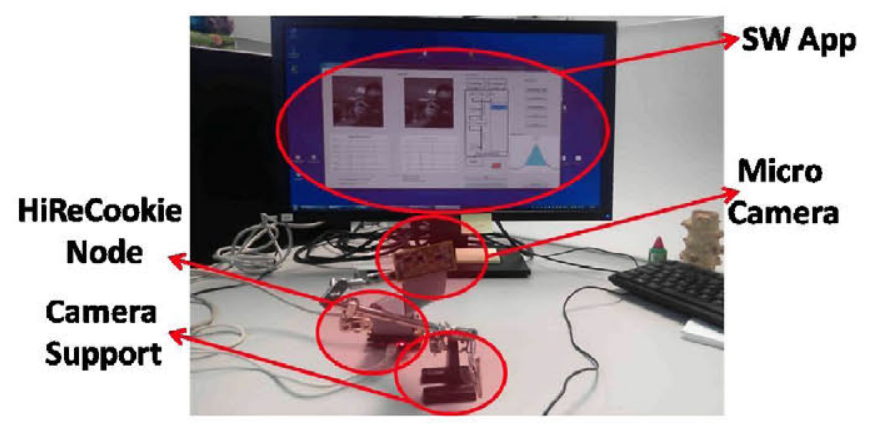

Fig. 1. Demonstration setup: camera, HiReCookie node and application

- It will be possible to change the level of noise in the image to check how the system reacts.

- It will be possible to move the camera freely to observe how the system reacts removing the noise and detecting edges in real time.

\section{ACKNOWLEDGMENT}

The authors would like to acknowledge the support of ARTEMIS JU and the Spanish Ministry of Industry and Commerce for the WSN-DPCM project under grant ART010000-2011-1.

The authors would also like to thank the Spanish Ministry of Education, Culture and Sport for their support under the FPU grant program.

\section{REFERENCES}

[1] Rodriguez, A.; Valverde, J.; de la Torre, E.; Riesgo, T., "Dynamic management of multikernel multithread accelerators using Dynamic Partial Reconfiguration," Reconfigurable and Communication-Centric Systems-on-Chip (ReCoSoC), 2014 9th International Symposium on , vol., no., pp.1,7, 26-28 May 2014

[2] Valverde, J.; Rodriguez, A.; Camarero, J.; Otero, A.; Portilla, J.; de la Torre, E.; Riesgo, T., "A dynamically adaptable bus architecture for trading-off among performance, consumption and dependability in Cyber-Physical Systems," Field Programmable Logic and Applications (FPL), 2014 24th International Conference on , vol., no., pp.1,4, 2-4 Sept. 2014

[3] Valverde, J.; Otero, A.; Lopez, M.; Portilla, J.; de la Torre, E.; Riesgo, T., "Using SRAM Based FPGAs for Power-Aware High Performance Wireless Sensor Networks," Sensors, vol. 12, no. 3, pp. 2667-2692, 2012

[4] Valverde, J.; de la Torre, E.; Portilla, J., FPGA-Based High Performance Wireless Sensor Node, Lambert Academic Publishing (LAP), 2014 